\title{
Prevention and treatment of diabetes with resveratrol in a non-obese mouse model of type 1 diabetes
}

\author{
S.-M. Lee $\cdot$ H. Yang $\cdot$ D. M. Tartar • B. Gao $・$ X. Luo $・$ \\ S. Q. Ye • H. Zaghouani • D. Fang
}

Received: 15 October 2010 /Accepted: 23 December 2010/Published online: 22 February 2011

(C) Springer-Verlag 2011

\begin{abstract}
Aims/hypothesis We recently found that activation of the type III histone deacetylase sirtuin 1 suppresses $\mathrm{T}$ cell immune responses. Here we sought to determine the therapeutic potential of the sirtuin 1 activator resveratrol in the treatment of diabetes in the NOD mouse model of type 1 diabetes and the mechanisms underlying such potential.

Methods NOD mice were fed or subcutaneously injected with resveratrol and evaluated for development of diabetes. Splenocytes from resveratrol-treated and control mice were analysed by gene array. The altered expression of inflammatory genes induced by resveratrol was validated and the role of changed gene expression in prevention of diabetes was determined.
\end{abstract}

Electronic supplementary material The online version of this article (doi:10.1007/s00125-011-2064-1) contains supplementary material, which is available to authorised users.

S.-M. Lee $(\bowtie) \cdot$ H. Yang $\cdot$ B. Gao $\cdot$ D. Fang $(\bowtie)$

Department of Pathology, Northwestern University Feinberg

School of Medicine,

303 E. Chicago Ave,

Chicago, IL 60612, USA

e-mail: leesangm@Northwestern.edu

e-mail: FangD@Northwestern.edu

S.-M. Lee

Division of Biotechnology, College of Environmental and

Bioresource Sciences, Chonbuk National University,

Jeonbuk, South Korea

D. M. Tartar $\cdot$ H. Zaghouani

Department of Molecular Microbiology and Immunology,

University of Missouri,

Columbia, MO, USA
Results Resveratrol administration potently prevented and treated type 1 diabetes in NOD mice. Gene array analysis indicated a dramatic decrease in expression of $C$ cr 6 , which encodes chemokine (C-C motif) receptor (CCR) 6, in the splenocytes from resveratrol-treated mice. CCR6 abundance on IL-17-producing cells and CD11b ${ }^{+} \mathrm{F} 4 / 80^{\mathrm{hi}}$ macrophages was inhibited by resveratrol treatment. Interestingly, $\mathrm{CCR}^{+}$IL-17-producing cells and $\mathrm{CD} 11 \mathrm{~b}^{+} \mathrm{F} 4 / 80^{\mathrm{hi}}$ macrophages accumulated in the spleens and pancreatic lymph nodes, but their presence in the pancreas was reduced, suggesting that resveratrol blocks their migration from peripheral lymphoid organs to the pancreas. Indeed, the migration of splenocytes toward media containing chemokine (C-C motif) ligand 20 (CCL20) was impaired by

X. Luo

Department of Medicine, Northwestern University Feinberg

School of Medicine,

Chicago, IL, USA

S. Q. Ye

Department of Medical Research, University of Missouri Kansas City School of Medicine,

Kansas City, MO, USA

S. Q. Ye

Department of Pediatrics, University of Missouri Kansas City

School of Medicine,

Kansas City, MO, USA

S. Q. Ye

Department of Biomedical and Health Informatics, University of Missouri Kansas City School of Medicine,

Kansas City, MO, USA 
resveratrol treatment. CCL20 peptides, which block CCR6 binding to CCL20, inhibited development of type 1 diabetes.

Conclusions/interpretation Inhibition of CCR6-mediated migration of inflammatory cells by resveratrol may provide a powerful approach for treatment of type 1 diabetes and possibly of other inflammatory diseases.

Keywords C-C chemokine receptor $6 \cdot \mathrm{CCR} 6 \cdot$ Disease prevention $\cdot$ Resveratrol Type 1 diabetes

$\begin{array}{ll}\text { Abbreviations } \\ \text { APC } & \text { Antigen-presenting cell } \\ \text { ALT } & \text { Alanine aminotranferease } \\ \text { AST } & \text { Aspartate aminotransferase } \\ \text { CCL20 } & \text { C-C Chemokine ligand 20 } \\ \text { CCR } & \text { Chemokine (C-C motif) receptor } \\ \text { EAE } & \text { Experimental autoimmune encephalomyelitis } \\ \text { FOX } & \text { Forkhead box } \\ \text { FSC } & \text { Forward scatter } \\ \text { NF-kB } & \text { Nuclear factor } \text { kB } \\ \text { PI3K } & \text { Phosphoinositide 3-kinase } \\ \text { SSC } & \text { Side scatter } \\ \text { Th } & \text { T helper } \\ \text { Treg } & \text { Regulatory T cell }\end{array}$

\section{Introduction}

Resveratrol (trans-3,4',5-trihydroxystilbene), a compound of the polyphenol group that is found in various fruits and vegetables, and is abundant in grapes [1], has recently emerged as a potential therapeutic reagent for many diseases, such as type 2 diabetes [2], cardiovascular heart diseases [3], cancers [4] and disease associated with ageing [5]. It has been suggested that resveratrol has immunosuppressive activities and thus therapeutic potential in the treatment/prevention of autoimmune diseases [6]. Feeding with resveratrol has recently been found to protect mice from experimental autoimmune encephalomyelitis (EAE) $[7,8]$. The root extract of the weed Polygonum cuspidatum, an important constituent of Chinese and Japanese folk medicines, is the richest known source of resveratrol and has been used for the treatment of lupus and rheumatoid arthritis for many years. In addition, recent studies have demonstrated that resveratrol has a protective effect on allograft rejection in mice $[9,10]$.

The possible mechanisms for the pharmacological effects of resveratrol, although extensively analysed, remain largely unknown. Administration of resveratrol to mice inhibits body weight and liver weight gain induced by highenergy diet, prevents pancreatic damage and extends the animals' lifespan [11]. These therapeutic activities of resveratrol are associated with: (1) increased insulin sensitivity; (2) reduced IGF-1 levels; (3) increases in AMP-activated protein kinase and peroxisome proliferator-activated receptor-c coactivator 1a activity; (4) increased mitochondrial number; and (5) improved motor function [12, 13]. By suppressing inhibitor of nuclear factor- $k \mathrm{~B}(\mathrm{NF}-\mathrm{kB})(\mathrm{I} \kappa \mathrm{B})$ kinase (IKK)-mediated phosphorylation of $\mathrm{I} \kappa \mathrm{B}$, resveratrol inhibits NF- $\mathrm{KB}$ and prevents overproduction of 12-O-tetradecanoylphorbol-13-acetateinduced cyclooxygenase-2, which is considered a major mechanism of its anti-inflammatory activities [14]. Resveratrol has also been found to have inhibitory effects on many other protein and lipid kinases, such as mitogen-activated protein kinase, plasma creatine kinase, Src family tyrosine kinases and phosphoinositide 3-kinase (PI3K). Resveratrol is well-known as an activator of sirtuin 1, an NADdependent sirtuin family deacetylase [15-17]. We recently demonstrated that sirtuin 1 suppresses $\mathrm{T}$ cell immunity in mice by deacetylating c-Jun, an activating protein-1 family transcription factor, and that resveratrol inhibits c-Jun acetylation in connection with its suppressive effect on $\mathrm{T}$ cell activation [18]. Adenovirus-mediated overproduction of sirtuin 1 protects pancreatic beta cells against cytokine toxicity by suppressing the NF-kB pathway [19].

Resveratrol has been found to prevent inflammation in mice by suppressing NF-kB-mediated production of inflammatory cytokines and by inducing apoptosis $[7,8]$. In this report, we found that resveratrol inhibits chemokine (C$\mathrm{C}$ motif) receptor (CCR) 6 production on immune cells, leading to minimal pancreatic islet infiltration and suppression of diabetes in the NOD mouse model of type 1 diabetes.

\section{Methods}

Mice NOD $\left(\mathrm{H}_{-} 2^{\mathrm{g} 7}\right)$, NOD.BDC2.5 and NOD/SCID mice were purchased from Jackson Laboratory (Bar Harbor, ME, USA) and used according to the guidelines of the Northwestern University Animal Care and Use Committee.

Diabetes assessment Assessment of blood glucose levels was by test strips and a monitoring system (Accu-Chek Advantage; Pompano Beach, FL, USA). A mouse was considered hyperglycaemic or diabetic when the blood glucose level reached $9-14$ or $16 \mathrm{mmol} / \mathrm{l}$, respectively, for two consecutive weeks.

Resveratrol treatment For feeding, female NOD mice were given $250 \mathrm{mg} / \mathrm{kg}$ resveratrol or Mega resveratrol (Sigma, St Louis, MO, USA) of $99 \%$ purity as confirmed by HPLC in $100 \mu \mathrm{l}$ PBS. Administration was daily by gavage from 
8 weeks of age. For injections, NOD mice were injected every other day with $25 \mathrm{mg} / \mathrm{kg}$ (in $100 \mu \mathrm{l}$ PBS) subcutaneously. The mice were monitored for blood glucose levels once a week up to 32 weeks of age.

Histology Pancreas histology was assessed as described [20]. Pancreases were removed from NOD females, fixed in $10 \%$ formalin and embedded in paraffin. Sections of $7 \mu \mathrm{m}$ thickness were cut at $100 \mu \mathrm{m}$ intervals to prevent double-counting of the same islet. Two sections per pancreas were stained with haematoxylin and eosin and analysed by light microscopy. Insulitis scoring was performed according to the following criteria: severe insulitis, $50 \%$ or more of the islet area infiltrated; mild insulitis, $<50 \%$ of the islet area infiltrated; peri-insulitis, infiltration restricted to the periphery of islets; no insulitis, absence of cell infiltration.

For immunochemistry analysis, paraffin-fixed pancreatic tissue sections were incubated overnight at $4{ }^{\circ} \mathrm{C}$ with antiinsulin (Abcam, Cambridge, MA, USA) and anti-F4/80 antibodies, and with the Alexa 594 goat anti-rabbit and Alexa 488 goat anti-rat IgG antibodies (Invitrogen, Carlsbad, CA, USA) at room temperature for $1 \mathrm{~h}$. After five extensive washings with $0.1 \%$ Triton X100-containing PBS, sections were stained for $5 \mathrm{~min}$ with $0.5 \mathrm{mg} / \mathrm{ml}$ DAPI followed by three additional washings. Tissue sections were visualised with fluorescent microscope.

Flow cytometry For cell surface protein staining, cells were removed from spleens and pancreatic lymph nodes, and incubated with FITC, phycoerythrin (PE)-, Cy5- or antigenpresenting cell (APC)-conjugated antibodies against mouse CD4, CCR4, CCR6, CCR7, CD11b, CD80, CD86, MHCI and $\mathrm{MHCII}$ in combinations as described. Subsequently, the cells were washed and fixed with $1 \%$ formaldehyde for $20 \mathrm{~min}$ at room temperature and then analysed. Cell apoptosis was analysed by annexin $\mathrm{V}$ staining as reported [21]. All antibodies were purchased from Biolegend (San Diego, CA, USA).

For intracellular cytokine analysis of IFN- $\gamma$ and IL-17, the splenic cells $\left(2 \times 10^{6}\right.$ cell $\left./ \mathrm{ml}\right)$ were stimulated for $4 \mathrm{~h}$ with $10 \mathrm{ng} / \mathrm{ml}$ phorbol myristate acetate and ionomycin $(1 \mu \mathrm{mol} / \mathrm{l})$ in the presence of brefeldin A $(10 \mu \mathrm{g} / \mathrm{ml})$ to block cytokine secretion and facilitate intracellular accumulation. The antibodies used were peridinin chlorophyll protein-anti-CD4, phycoerythrin-anti-IFN- $\gamma$ and APC-antiIL-17 (BD Biosciences, San Diego, CA, USA). Isotypematched controls were included in all experiments. Events were collected on FACScan, FACSCanto II (San Jose, CA, USA) or CyanADP (Daco, Carpinteria, CA, USA) flow cytometers and analysed with CellQuest software (Becton Dickinson, Franklin Lakes, NJ, USA) or Summit (Beckman-Coulter, Fullerton, CA, USA).
Adoptive transfer experiments For disease transfer, $1 \times 10^{7}$ freshly isolated splenocytes from resveratrol-treated or PBS-treated mice were injected into 6-8-week-old NOD/ SCID mice. Blood glucose levels were monitored weekly in the first 3 weeks after transfer and then every other day.

Splenocyte migration assay Freshly isolated splenocytes were incubated on 24-well collagen-coated, polyester membrane Transwell inserts of $6.5 \mathrm{~mm}$ diameter and $3.0 \mu \mathrm{m}$ pore size (Corning, Lowell, MA, USA). The confluent inserts were transferred to fresh wells containing $600 \mu \mathrm{l}$ of TEM buffer with $10 \mathrm{ng} / \mathrm{ml}$ recombinant mouse CC chemokine ligand 20 (CCL20) (R\&D Systems, Minneapolis, MN, USA). Cells were allowed to transmigrate for $3 \mathrm{~h}$ at $37^{\circ} \mathrm{C}$. These experiments were performed in triplicate, with migration across the basal in vitro bloodbrain barrier without added chemokine serving as spontaneous migration.

Real-time PCR analysis RNA was isolated from spleens, pancreatic lymph nodes or pancreases using TRIzol reagent (Invitrogen) and was reverse-transcribed using Moloney murine leukaemia virus reverse transcriptase (Invitrogen). Quantitative real-time RT-PCR was performed on a sequence detection system (Prism 7000; Applied Biosystems, Foster City, CA, USA). $\beta$-Actin gene was used as a reference for sample normalisation. Primers and probes for the following genes including Ifn- $\gamma$ (also known as Ifng), Il10, Ill7 (also known as Il17a), Cd11c (also known as Itgax), Cd11b (also known as Itgam), F4/80 (also known as Emr 1), Ccr4 and Ccr6 were purchased as assays-ondemand (Applied Biosystems, Bedford, MA, USA). Standard amplification protocol was used following the manufacturer's description.

Statistical analysis The $\chi^{2}$ test was used for incidence of diabetes analysis among experimental and control groups. For the rest of the experiments, $p$ values were calculated with the two-tailed Student's unpaired $t$ test.

\section{Results}

Resveratrol protects NOD mice from diabetes To investigate the effects of resveratrol on type 1 diabetes mellitus, NOD mice were fed a daily dose of $250 \mathrm{mg}$ resveratrol per kilogram of body weight, beginning at week 8 of age as recently reported [7]. Disease onset was significantly delayed and $30 \%$ of the mice remained free of type 1 diabetes mellitus for the duration of the experiment (Electronic supplementary material [ESM] Fig. 1). A regimen using subcutaneous injection of $25 \mathrm{mg}$ resvera- 


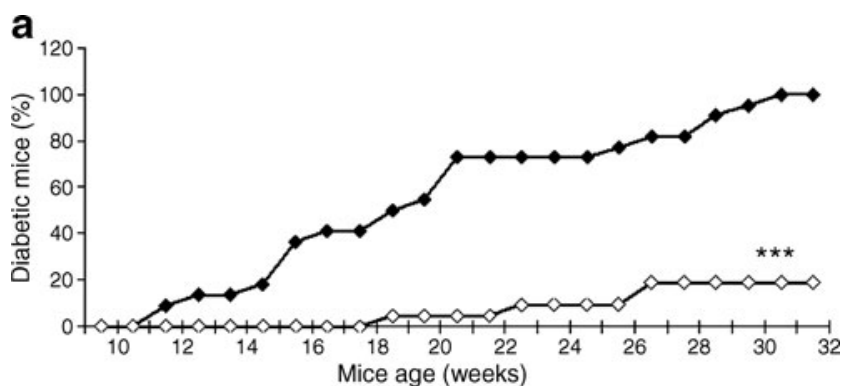

b



d

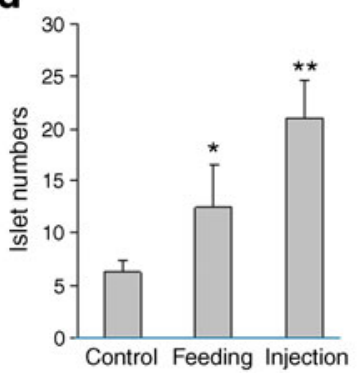

f

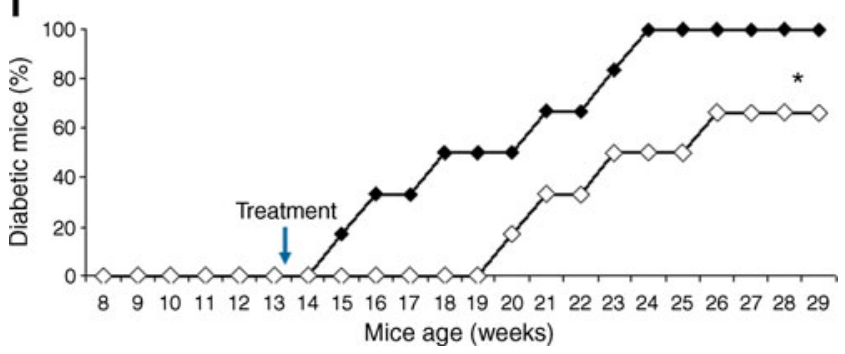

trol/kg every other day was even more effective, as more than $80 \%$ of the mice were protected from type 1 diabetes mellitus (Fig. 1a, ESM Fig. 1c), a finding that correlates well with the suggestion that the oral route is less efficient due to low absorption of the drug [15-17]. Analysis of the serum levels of the liver enzymes alanine aminotranferease (ALT) and aspartate aminotransferase (AST) did not reveal any liver damage in treated mice. The average ALT level in control mice and AST level in all groups were slightly higher than normal, possibly because some mice were diabetic [22]. Serum creatinine levels were all in the normal ranges in mice of all three groups, indicating that resveratrol treatment did not harm kidney functions (ESM Table 1).
Fig. 1 Resveratrol protects NOD mice against development of autoimmune diabetes. a NOD mice were injected subcutaneously every other day with resveratrol at $25 \mathrm{mg} / \mathrm{kg}$ (white diamonds) or PBS (black diamonds). Blood glucose levels were monitored weekly; percentages of diabetic mice are shown $(n=21)$. b Pancreases were isolated from control PBS-treated or (c) resveratrol-treated mice, and pancreas sections stained with haematoxylin and eosin. Representative images are shown. $\mathbf{d}$ The islet numbers in each pancreas section were counted and levels of inflammation quantified (e). Values are presented as mean $\pm \mathrm{SD}$ (error bars) and represent data from 12 mice from each group. ${ }^{*} p<0.05$, ${ }^{* *} p<0.01$. Black bars: no insulitis; grey bars: peri-insulitis; hatched bars, mild insulitis; horizontally striped bars, severe insulitis. f NOD mice were treated with resveratrol (white diamonds) or PBS (black diamonds) from the age of 13 weeks, when most have insulitis $(n=6)$. Blood glucose levels were monitored weekly, with percentage of diabetic mice shown. ${ }^{*} p<0.05$. Mice in (d) and (e) were treated by feeding $(250 \mathrm{mg} / \mathrm{kg})$ or injection; the rest of figure parts show data from mice treated with resveratrol injection

To further investigate the preventive roles of resveratrol against type 1 diabetes mellitus, we performed histological analysis of islet infiltration upon treatment with resveratrol at a time point when $50 \%$ of the control mice manifested diabetes. In the PBS-treated mice, most of the islets exhibited intra-insulitis, while the mice receiving subcutaneous resveratrol had mild insulitis with most islets remaining healthy. Similarly, recipients of oral resveratrol also had mild insulitis in comparison with control mice (Fig. 1b-e). Enumeration of the islets indicated that resveratrol-treated animals had a significantly greater number of total islets than PBS-treated control mice (Fig. 1d). Indeed, resveratrol-treated groups had a higher percentage of insulitis-free islets $(69 \%$ for injection and $31 \%$ for feeding delivery vs $16 \%$ ) and a significantly reduced number of islets with mild $(10 \%$ for injection and $25 \%$ for feeding vs $34 \%)$ or severe insulitis $(1 \%$ for subcutaneous injection and $14 \%$ for oral feeding vs $21 \%$ ) relative to PBS-treated mice (Fig. 1e). Further analysis of the effects of resveratrol indicated that lymphocytes from resveratrol-treated mice were less pathogenic. When total splenocytes from resveratrol-treated and control mice were adoptively transferred into NOD/SCID mice, only $20 \%$ (one of five) became diabetic in the resveratrol-treated group, while $60 \%$ (three of five) of the control mice became diabetic (ESM Fig. 2). Furthermore, all five control mice developed diabetes at 5 weeks after transfer, while two of five resveratrol-treated mice remained non-diabetic at this time-point (ESM Fig. 2). These results indicate that resveratrol prevents spontaneous diabetes mellitus in the NOD mouse model of type 1 diabetes and may reverse type 1 diabetes if given at advanced stages of the disease.

To investigate whether resveratrol can reverse diabetes, we initiated resveratrol treatment at week 13 of age, when essentially all NOD mice have insulitis and about 10-20\% had hyperglycaemia. While about $50 \%$ of the control mice 
had diabetes at week 19 of age, all resveratrol-treated mice remained healthy at that time-point. Morover, two of six resveratrol-treated mice were still protected from diabetes at 29 weeks of age, whereas all control animals had diabetes, suggesting that resveratrol has protective effects even after insulitis development in NOD mice (Fig. 1f).

The effect of resveratrol on $T$ cell activation and cytokine production in treated NOD mice Intracellular staining analysis revealed a decrease in IFN- $\gamma$-producing $\mathrm{CD} 4^{+}$ and $\mathrm{CD}^{+} \mathrm{T}$ cells in the spleens of resveratrol-treated mice, suggesting that resveratrol may prevent diabetes by suppressing IFN- $\gamma$ production (Fig. $2 \mathrm{a}-\mathrm{c}$ ). Unexpectedly, the percentage of IL-17-producing $\mathrm{CD} 4^{+} \mathrm{T}$ cells was significantly increased in the spleens of resveratrol-treated mice (Figs 2c, 3c, ESM Fig. 3). Levels of IFN- $\gamma-$ and IL-17producing cells increased with age and diabetes development, as we observed significant higher percentages in diabetic mice than in young healthy NOD mice (ESM Fig. 3a). In addition, we observed significantly increased IL-10-producing $\mathrm{CD}^{+}{ }^{+} \mathrm{T}$ cell population in treated mice when compared with control mice (Fig. 2c). Similarly, realtime RT-PCR analysis revealed increased $I l 17$ and $I l 10$ mRNA levels, but decreased Ifn- $\gamma$ mRNA expression in
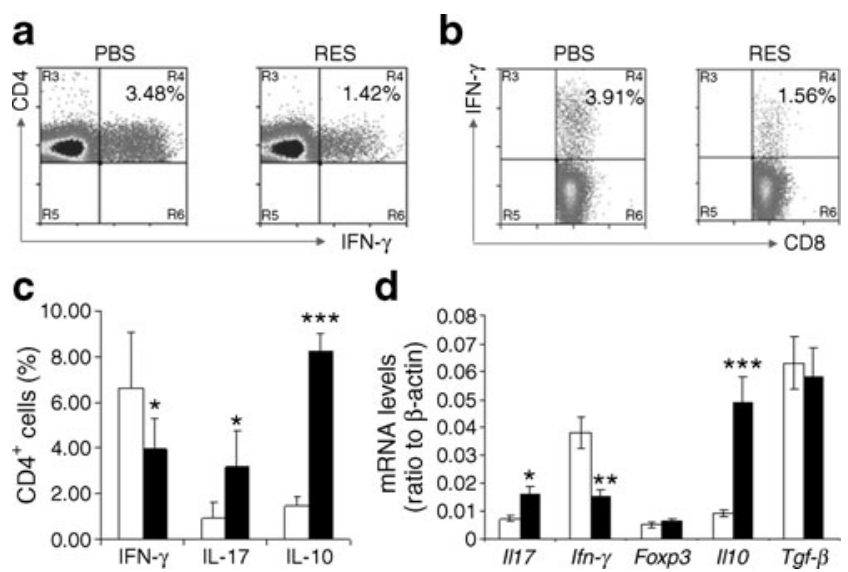

Fig. 2 The effects of resveratrol on cytokine production by $\mathrm{CD}^{+} \mathrm{T}$ cells in NOD mice. a, b Splenocytes from PBS- or resveratrol-treated (RES) mice (20 weeks old) were cultivated with phorbol myristate acetate plus ionomycin for $4 \mathrm{~h}$ in the presence of $10 \mu \mathrm{g} / \mathrm{ml}$ brefedlin A. IFN- $\gamma$ levels in $\mathrm{CD}^{+}$(a) and $\mathrm{CD}^{+}$(b) $\mathrm{T}$ cells were analysed by intracellular staining. $\mathbf{c}$ The average percentages of IFN- $\gamma$, IL-17 and IL- $10^{+} \mathrm{CD} 4 \mathrm{~T}$ cells as analysed by intracellular staining and flow cytometry in spleens from PBS (white bars) and resveratrol-treated (black bars) mice. Values are presented as mean \pm SD (error bars) and represent data from at least 12 mice in each group. d Pancreatic lymph nodes of resveratrol- (black bars) and PBS-treated (white bars) mice were isolated. Cells were pooled from five mice in each group and total RNA was isolated. The expression of Ill1, Ifn- $\gamma$, Foxp $3, I l 10$ and $T g f-\beta$ was analysed by real-time PCR. The relative levels indicate ratios of indicated genea to $\beta$-actin control. Values are presented as mean \pm SD (error bars) and represent data from three experiments. ${ }^{*} p<0.05, * * p<0.01, * * * p<0.001$ pancreatic lymph nodes from resveratrol-treated mice (ESM Fig. 3c). However, levels of Foxp 3 and $T g f-\beta$ (also known as $T g f b 1)$ mRNA were indistinguishable between PBS- and resveratrol-treated mice (Fig. 2d). Since IFN- $\gamma$ has suppressive functions in T helper (Th)17 polarisation [23, 24], the increase in IL-17-producing $\mathrm{T}$ cells may have been partially due to the decreased number of IFN- $\gamma$ in spleen and pancreatic lymph nodes from resveratrol-treated mice. In fact, a recent elegant study demonstrated that absence of IFN- $\gamma$ in $\mathrm{CD}^{+} \mathrm{T}$ cells resulted in augmented Th2 and Th17 differentiation and exacerbated tissue damage in lung and skin [25].

$\mathrm{CD}^{+}$regulatory T cells (Tregs) have been considered one of the key players in suppression of autoimmune diseases such as type 1 diabetes mellitus. We thus asked whether resveratrol prevents type 1 diabetes mellitus in NOD mice by upregulating Tregs. However, the percentages of forkhead box (FOX)P3 ${ }^{+}$Tregs in spleen, pancreatic lymph nodes and pancreas were indistinguishable between resveratrol-treated and control mice (ESM Fig. 4a). The cell surface expression of suppressive molecules, including cytotoxic $\mathrm{T}$ lymphocyte antigen 4, glucocorticoid-induced TNF receptor familyrelated gene and TGF- $\beta$, on Tregs was not affected by resveratrol treatment (ESM Fig. 4c). $\mathrm{FOXP}^{+} \mathrm{CD}^{+} \mathrm{T}$ cells have recently been characterised as a new subset of Tregs, which has been shown to protect rats from diabetes $[26,27]$. However, resveratrol appears not to affect $\mathrm{CD} 8^{+}$Tregs in NOD mice, as the percentages of $\mathrm{CD}^{-}{ }^{-} \mathrm{FOXP} 3^{+}$cells, which presumably includes $\mathrm{CD} 8^{+} \mathrm{FOXP} 3^{+}$Tregs, were indistinguishable between resveratrol-treated and control mice (ESM Fig. 4b). In addition, treatment of mice with resveratrol did not affect in vivo $\mathrm{T}$ cell activation as analysed by cell surface expression of CD44, CD62L and CD69 (ESM Fig. 5a, b), nor did it affect the Fas ligand levels and apoptosis of $\mathrm{T}$ and $\mathrm{B}$ cells in the spleen (ESM Fig. 5c, d). Therefore, resveratrol appears not to affect Treg development or function, or to suppress $\mathrm{T}$ cell activation in mice.

Resveratrol downregulates Ccr6 expression To investigate how resveratrol protects NOD mice from diabetes, we performed inflammatory gene array chip analysis of the expression profile of genes associated with inflammatory autoimmune diseases in resveratrol- and PBS-treated mice. The findings clearly demonstrate that $C$ cr6 expression was significantly downregulated by resveratrol, while the expression of other chemokine receptor genes remained at similar levels in both animal groups (Fig. 3a). To validate the array data, we analysed CCR6 protein expression on the surface of $T$ cells from resveratrol-treated mice and indeed confirmed a significant reduction on $\mathrm{T}$ cells from the spleen and pancreatic lymph nodes of resveratrol-treated mice compared with PBS-treated controls (Fig. 3b). This in vivo Ccr6 downregulation in $\mathrm{T}$ cells by resveratrol was further 
a

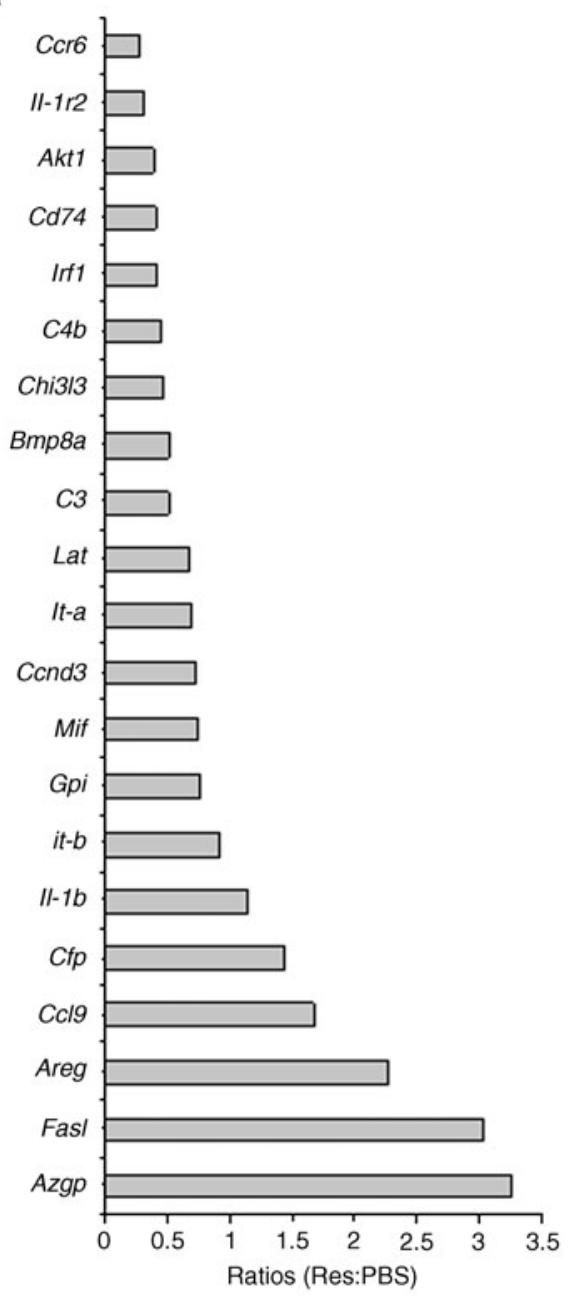

e

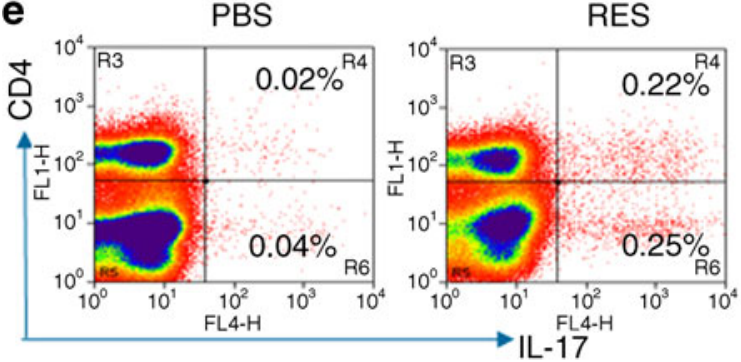

Fig. 3 Reduced CCR6 levels in splenocytes from resveratrol-treated mice. a Total RNA was isolated from splenocytes of PBS- or resveratrol-treated (RES) mice. mRNA was reverse-transcribed into cDNA, labelled with biotin and analysed by inflammation array kit (SAbiosciences, Frederick, MD, USA). Each number represents the ratio of gene expression in resveratrol-treated splenocyte to PBStreated controls. Gpi (also known as Gpil), Azgp (also known as Azgp 1). b Levels of CCR6 on $\mathrm{CD}^{+}{ }^{+} \mathrm{T}$ cells from spleen (black) and pancreatic lymph nodes (blue) were analysed by flow cytometry. FL2, forward light scatter 2; PE-A, phycoerythrin. $\mathbf{c} \mathrm{CD}^{+}$and $\mathrm{CD}^{+} \mathrm{T}$ cells, and $\mathrm{F} 4 / 80^{+}$macrophages were isolated from the spleens of resveratrol- (black bars) or control PBS-treated (white bars) mice by magnetic antibody cell sorting (MACS). Total RNA was isolated and


Ccr6 mRNA transcription levels were determined by real-time PCR using $\beta$-actin as control. Values are ratios Ccr6: $\beta$-actin, and are shown as mean \pm SD (error bars) from five pairs of mice. ${ }^{* *} p<0.01$, $* * * p<0.001$. d Total T cells and $\mathrm{F} 4 / 80+$ macrophages were isolated with MACS and lysed. The levels of CCR6 (top panel) and $\beta$-actin (bottom panel) were detected by western blotting. Lane 1, T cells from control mice; lane 2, T cells from resveratrol-treated mice; lanes 3 and 4, macrophages from control diabetic and resveratrol-treated mice, respectively. e IL-17-positive cells in splenocytes from resveratrol- or PBS-treated mice were analysed by intracellular staining and flow cytometry. f CCR6 levels on IL-17-positive cells were analysed by flow cytometry. Numbers represent fluorescence intensities. FL1-H, forward light scatter $1-\mathrm{H}$ 
confirmed by real-time PCR analysis and western blotting (Fig. 3c, d). The controls, CCR4 and CCR7, were comparable between resveratrol- and PBS-treated mice (ESM Fig. 6). However, analysis of chemokine gene expression showed no measurable effect of resveratrol treatment on any of the chemokines tested. Given that CCR6 is involved in trafficking of autoimmune lymphocytes to inflammatory tissues and organs [28, 29], downregulation of Ccr6 expression in this case may be one of the mechanisms by which resveratrol prevents type 1 diabetes in mice.

Reduced CCR6 expression in Th17 and CD11b F4/80 hi macrophages in resveratrol-treated mice It is well established that chemokine receptors are differentially expressed on subsets of effector and memory $\mathrm{T}$ cells, and provide specific trafficking in the steady state and in inflammation [30]. Given that CCR6 was reduced on splenic cells and that the number of IFN- $\gamma$-producing cells was diminished but that of Th17 cells increased, we determined whether CCR6 downregulation occurred in Th17 cells. This could explain disease prevention by resveratrol, especially because CCR6 has been found to play a critical role in the trafficking of effector Th17 cells to the central nervous system in humans with multiple sclerosis and in mice with EAE [29]. The findings revealed a significant reduction of in CCR6 expression on IL-17-producing $\mathrm{CD}^{+}$and $\mathrm{CD} 4^{-} \mathrm{T}$ cells from resveratrol-treated mice (Fig. 3e, f). Moreover, IL-17-producing cells accumulated in the spleen of resveratrol-treated mice (Fig. 2), while their presence in the pancreas was dramatically reduced as measured by $I l 17$ mRNA expression (ESM Fig. 3c). These results suggest that reduced CCR6 expression on Th17 cells leads to reduced trafficking of the cells to the pancreas and their accumulation in the spleen.

During flow cytometry analysis, we noticed a reduction in the side scatter $(\mathrm{SSC})^{\mathrm{hi}}$ and forward scatter $(\mathrm{FSC})^{\text {low }}$ population of total splenocytes of diabetic mice and found that this population was protected by resveratrol treatment (ESM Fig. 7a). Further characterisation demonstrated that this population consisted of $\mathrm{CD}_{11} \mathrm{~b}^{+}$macrophages (ESM Fig. 7a). Splenocyte cellularity analysis indicated that the total $\mathrm{CD} 11 \mathrm{~b}^{+}$macrophage numbers in diabetic mice were reduced and that resveratrol treatment prevented this macrophage loss in the spleen (ESM Fig. 8). Interestingly, the levels of CCR6 on $\mathrm{SSC}^{\text {hi }}$ and $\mathrm{SSC}^{\text {low }} \mathrm{CD} 11 \mathrm{~b}^{+}$macrophages were reduced in NOD mice treated with resveratrol (ESM Fig. 7b). As a control, CCR4 expression on macrophages was not affected by resveratrol treatment (ESM Fig. $7 \mathrm{c})$.

$\mathrm{CD} 11 \mathrm{~b}^{+} \mathrm{F} 4 / 80^{\text {low }}$ and $\mathrm{CD} 11 \mathrm{~b}^{+} \mathrm{F} 4 / 80^{\text {hi }}$ cells were increased in spleens from resveratrol-treated mice (Fig. 4a). Resveratrol inhibited CCR6 expression on CD11b ${ }^{+} \mathrm{F} 4 / 80^{\mathrm{hi}}$,


pancreatic lymph nodes (Fig. 4b). In addition, we observed significant reductions in CD80 and CD86, but not MHCI and MHCII expression on macrophages from resveratroltreated mice (Fig. 4c, ESM Fig. 9). Collectively, our data suggest that resveratrol may protect mice from diabetes by suppressing migration and pathogenicities of $\mathrm{CD} 11 \mathrm{~b}^{+} \mathrm{F} 4 /$ $80^{\mathrm{hi}}$ macrophages.

Resveratrol impairs splenocyte trafficking toward CCL20 CCR6 is critical in the regulation of T effector cell trafficking toward sites where the CCR6 ligand, CCL20, is produced [31-36]. Haematoxylin and eosin staining analysis of pancreatic tissue sections revealed that resveratrol suppressed insulitis (Fig. 1b, c). Together with our finding of reduced CCR6 expression on T cells and macrophages, it is possible that resveratrol may inhibit islet inflammation by blocking migration of inflammatory cells to pancreas. Indeed, mRNA levels of Ccr6, but not Ccr4 and Ccl20 in the pancreas were reduced by $71 \%$ in resveratrol-treated compared with PBS-treated mice (Fig. 5a). In correlation with this reduction of CCR6 levels in pancreatic tissues of resveratrol-treated mice, mRNA levels of Cd11b (81\% reduction), Ifn- $\gamma$ (63\% reduction) and $I l 17$ (92\% reduction) were significantly reduced (Fig. 5b, ESM Fig. 3c), suggesting that resveratrol inhibits the migration of pathogenic $\mathrm{CD} 11 \mathrm{~b}^{+} \mathrm{F} 4 / 80^{\mathrm{hi}}$ macrophages and inflammatory $\mathrm{T}$ lymphocytes into the pancreas. We then performed an immunochemistry analysis to compare F4/80+ macrophages in the pancreases from resveratrol-treated and control mice. Similar to our results in haematoxylin and eosin staining, insulin staining further confirmed a significantly higher number of islets in the pancreases of resveratrol-treated mice. Interestingly, both in the islet and the interstitial area, $\mathrm{F} 4 / 80^{+}$cell numbers in the pancreases from resveratroltreated mice were dramatically reduced (Fig. 5d, e). Together with the increased $\mathrm{F} 4 / 80^{+}$macrophages in spleen and pancreatic lymph nodes (Fig. 4a, b), our results suggest that resveratrol may block pathogenic macrophage trafficking into the pancreases.

We therefore used an in vitro culture system and tested the migration ability of splenocytes from resveratrol-treated mice in a CCR6- and CCL20-dependent manner. Fewer than $3 \%$ of splenocytes from resveratrol-treated mice migrated into the compartment containing CCL20-rich medium. In contrast, the migration rate for splenocytes isolated from diabetic mice was $12 \%$ (Fig. 5c). Collectively, our data indicate that resveratrol inhibits inflammatory cell trafficking from peripheral lymphoid organs to the pancreas by suppressing CCR6 production.

Our finding that resveratrol prevented type 1 diabetes by suppressing production of CCR6, but not of its ligand CCL20, suggests an important role of CCR6-CCL20 crosstalk during 
Fig. 4 Characterisation of $\mathrm{CD}_{11} \mathrm{~b}^{+}$cells in resveratroltreated mice. a Cells from spleens and (b) pancreatic lymph nodes of PBS- or resveratrol-treated (RES) mice were analysed by their CD11b and F4/80 levels. c Expression of CD80 or (d) CCR6 on $\mathrm{CD} 11 \mathrm{~b}^{+} \mathrm{F} 4 / 80^{\text {low }}$ and $\mathrm{CD} 11 \mathrm{~b}^{+} \mathrm{F} 4 / 80^{\mathrm{hi}}$ was analysed by flow cytometry FL-4, forward light scatter 4
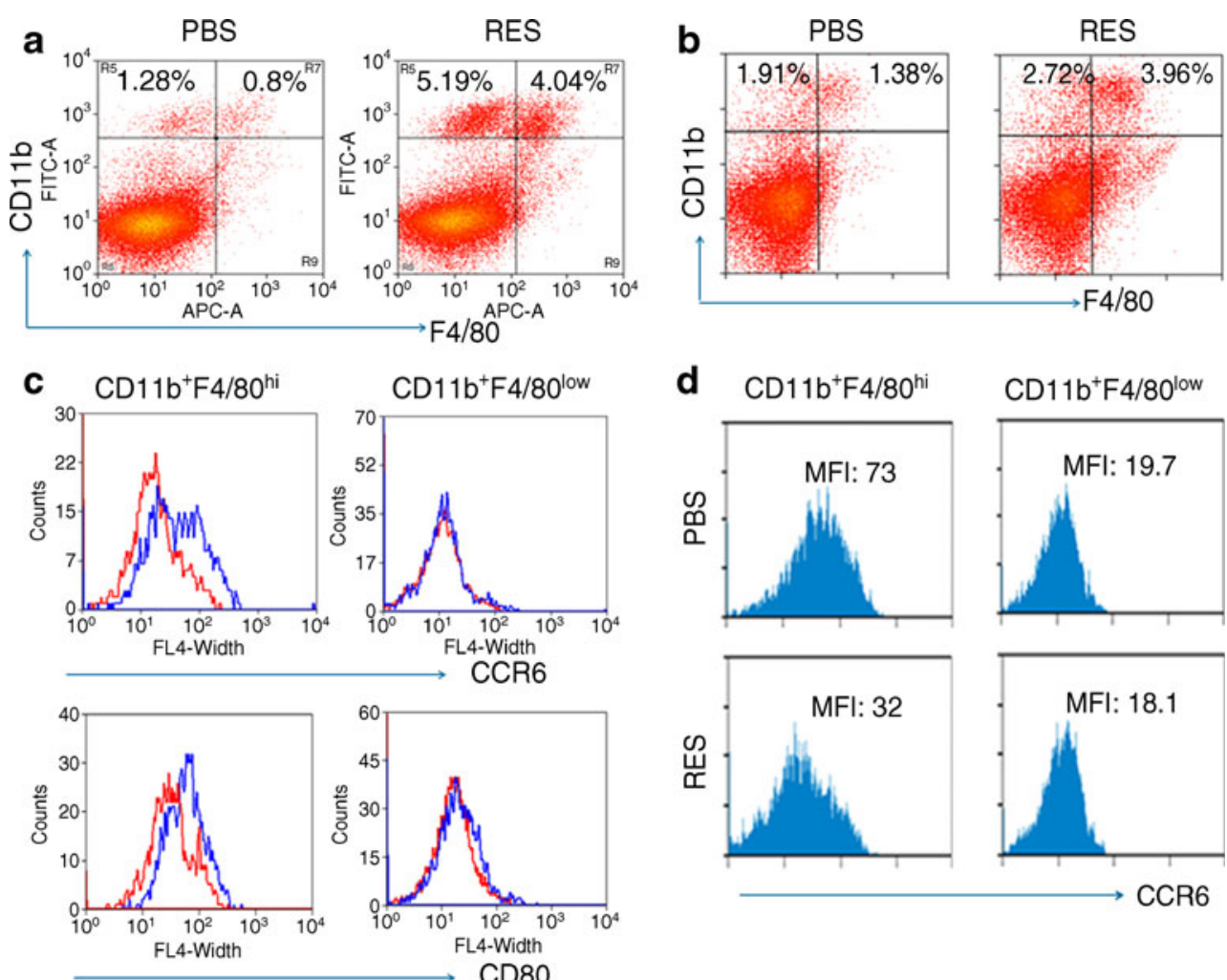

diabetes development. We therefore asked whether blocking of $\mathrm{CCR}^{+}$cell trafficking using CCL20 peptide might suppress diabetes in NOD/SCID mice that had been adoptively transferred with splenocytes from diabetic NOD mice. Indeed, administration of CCL20 peptide every other day significantly delayed disease onset and protected more than $70 \%$ mice from diabetes even at 40 days after transfer (Fig. 5f). Therefore, in the mouse model of type 1 diabetes used by us, blocking of CCR6-mediated migration of inflammatory cells prevents diabetes mellitus and resveratrol treats the condition by inhibiting CCR6 production.

\section{Discussion}

Type 1 diabetes is an autoimmune disease in which a selfdestructive immune process against the pancreatic beta cells leads to insulin deficiency. In this study, we found that administration of resveratrol, particularly by subcutaneous injection, in the NOD mouse model of type 1 diabetes delayed disease in more than $80 \%$ of the animals. Resveratrol downregulates CCR6 expression on multiple inflammatory cell types. This reduction of CCR6 expression appears to block the migration of pathogenic cells to the pancreas, since insulitis was dramatically inhibited by resveratrol treatment and splenocytes from resveratrol-treated mice have impaired ability to migrate towards CCL20, a CCR6 ligand [34].
Moreover, we found that CCL20 peptides, which have previously been shown to suppress EAE development in mice [24], displayed great efficacy in the treatment of diabetes in NOD/SCID mice that had received pathogenic splenocytes from diabetic mice.

CCR6 downregulation is an important mechanism of resveratrol in diabetes treatment and prevention. CCR6 has been found to be a critical mediator in pathogenic lymphocyte migration to sites of inflammation in different types of disease setting [37]. Rebodi et al. recently reported that CCR6 production is required for pathogenic Th17 cell migration to the central nervous system during EAE development in mice [29]. The involvement of Th17 cells in type 1 diabetes is still under debate [38-41], but recent studies have indicated that Th17 cell accumulation is involved in peripheral blood $\mathrm{T}$ cells from children with type 1 diabetes mellitus [42, 43]. Indeed, we found that CCR6 expression on $\mathrm{CD}^{+}$and $\mathrm{CD}^{-}$IL-17-producing cells was inhibited by resveratrol treatment. This CCR6 reduction seems likely to inhibit IL-17-producing cell migration to the pancreas, because $I l 17$ mRNA expression was decreased in pancreatic cells, but increased in the spleens of resveratrol-treated mice. It has been reported that $\mathrm{CD} 11 \mathrm{~b}^{+} \mathrm{F} 4 / 80^{\mathrm{hi}}$ macrophages retain lymphocytes in insulitis in NOD mice and that macrophage depletion inhibits diabetes development accompanied by pancreatic lymphocyte infiltration [37]. Resveratrol appears to suppress insulitis by blocking $\mathrm{CD} 11 \mathrm{~b}^{+} \mathrm{F} 4 / 80^{\mathrm{hi}}$ macrophage migration 




b

d

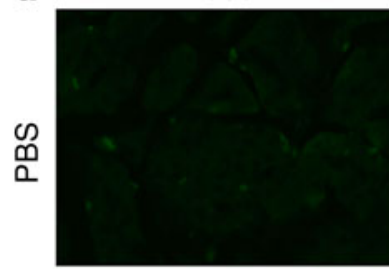

$\mathrm{F} 4 / 80$
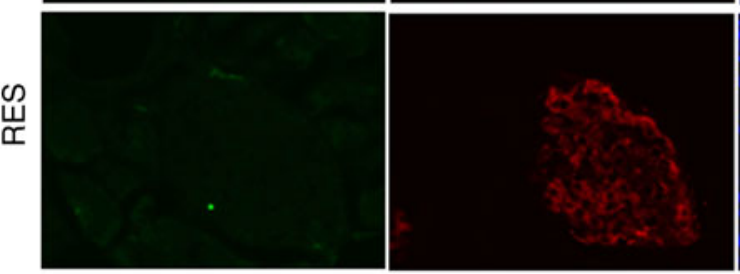

Insulin
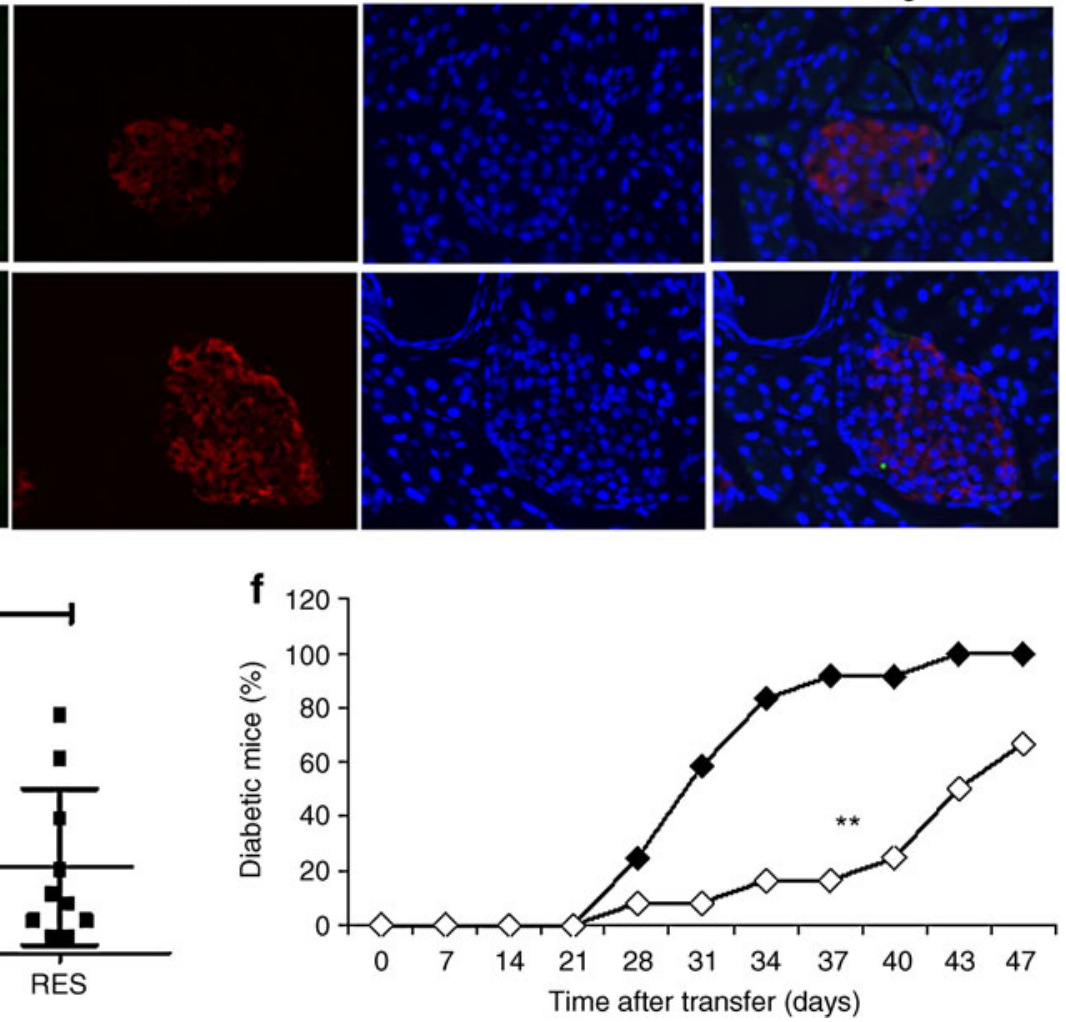

Fig. 5 Resveratrol suppresses splenocytes trafficking toward CCL20. a, b Total RNA was isolated from pancreatic tissues of resveratrol (black bars) and PBS-treated (white bars) mice. The expression of genes as indicated was analysed by real-time RT-PCR, with ratios to $\beta$-actin mRNA levels being shown. Values are mean \pm SD (error bars) and represent data from five pairs of mice in each group. c Freshly isolated splenocytes from PBS- or resveratrol-treated (RES) mice were cultured for $3 \mathrm{~h}$ in a chamber and their migration towards CCL20containing medium analysed. Values are mean $\pm \mathrm{SD}$ (error bars) and represent data from three experiments. d Pancreatic tissue sections were analysed by immunohistology with anti-insulin (red) and anti-

to the pancreas, because our data indicate that a reduction of $C d 11 \mathrm{~b}$ mRNA in pancreas is accompanied by an increase in percentage of $\mathrm{CD}_{11} \mathrm{~b}^{+}$macrophage in the spleens of resveratrol-treated mice. In addition, macrophages from resveratrol-treated mice had impaired ability to migrate towards CCL20 and administration of CCL20 peptides, which block $\mathrm{CCR}^{+}$cell trafficking in mice, suppressed
DAPI
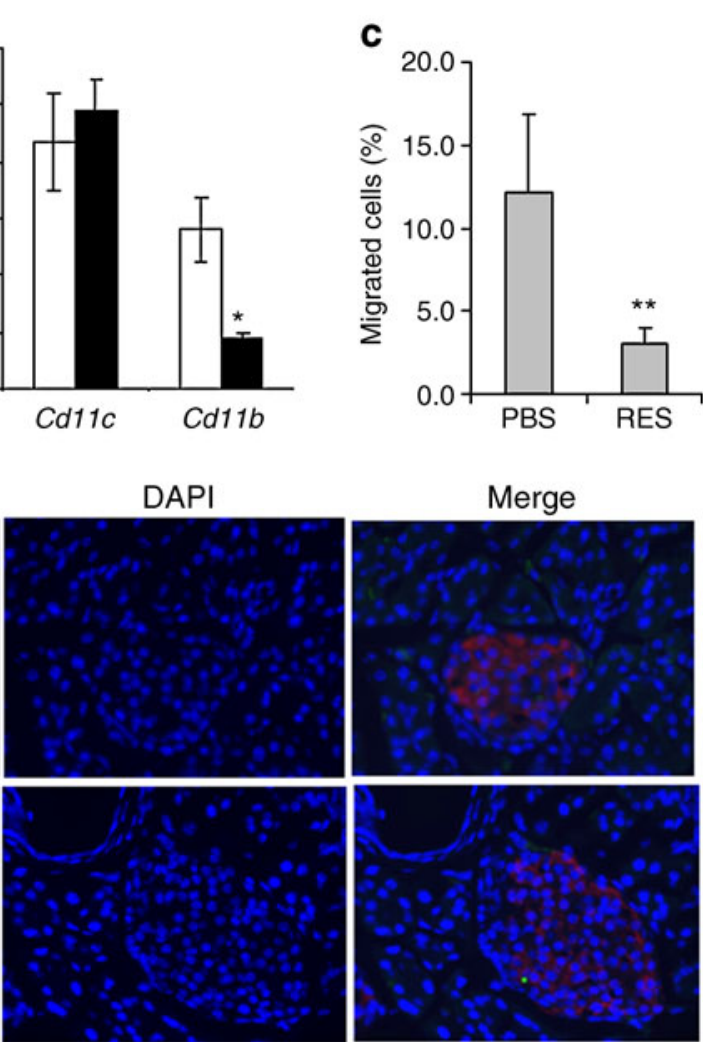

F4/80 (green) antibodies followed by DAPI staining for nuclear DNA (blue). e The numbers of F4/80+ cells per three scope field as shown (d) within each pancreatic section were counted. Pancreatic sections from ten mice in each group were analysed. $\mathbf{f}$ Total splenocytes from diabetic NOD mice were adoptively transferred into NOD/SCID mice. Recipients were treated with $100 \mu \mathrm{g}$ CCL20 peptide or control OVA peptide by i.p. injection every other day. The blood glucose levels were monitored weekly during the first 4 weeks after transfer and every 3 days thereafter. Percentages of diabetic mice are shown $(n=$ 12). Student's $t$ test was used for the statistical analysis, with $p$ values smaller than 0.05 considered significant. ${ }^{*} p<0.05,{ }^{* *} p<0.01$

diabetes in the NOD/SCID mouse model of type 1 diabetes when mice had been adoptively transferred with splenocytes from diabetic mice. Therefore, our studies reveal a novel mechanism in resveratrol treatment of type 1 diabetes and possibly of other types of autoimmune diseases

Resveratrol increases the level and activity of sirtuin 1, a mammalian $\mathrm{NAD}^{+}$-dependent histone deacetylase $[15,16$, 
44]. The deacetylase activity of sirtuin 1 is necessary for its suppressive activity in gene transcription. We have recently reported that sirtuin 1 inhibits $\mathrm{T}$ cell activation and that its upregulation is required for peripheral $\mathrm{T}$ cell tolerance [18]. Accumulating evidence indicates a function of resveratrol in cells and in mice that is independent of sirtuin 1 [45]. It has been reported that activation of many other proteins can be inhibited by resveratrol in cells. Many of these proteins are involved in regulation of immune response, such as NF- $\mathrm{KB}$, PI3K, FOXO3A and Janus kinase 1. Therefore, sirtuin 1 may merely contribute to a small portion of the in vivo activity of resveratrol.

Moreover, numerous previous studies have revealed that resveratrol affects many cell biological responses such as insulin sensitivity, gluco/lipotoxicity, endoplasmic reticulum stress, oxidative stress etc., all of which contribute to diabetes, in particular type 2 diabetes [46]. Our findings here define one of the immune suppressive functions of resveratrol in protecting mice from diabetes. We believe, however, that other mechanisms also contribute to the glucose-lowering functions of resveratrol in our model.

Our study suggests that resveratrol is a potential therapeutic and/or preventive reagent for type 1 diabetes in humans. Interestingly, the Food and Drug Administration of the USA has approved resveratrol for human use as a food supplement (GRN000224). The current 'mega doses' of resveratrol for humans is $500-1,000 \mathrm{mg}$ (about 7-15 mg/ $\mathrm{kg}$ body weight) per day, which is still much lower than the doses used in this study. Results from our study, using a much lower dose by injection $(25 \mathrm{mg} / \mathrm{kg})$, showed a greater efficacy of resveratrol in protecting mice from type 1 diabetes, reflecting the poor intestinal absorption of resveratrol. Therefore, more efforts are needed in the pharmaceutical studies to improve resveratrol absorption. Interestingly, more potent sirtuin 1 activators (about 1,000fold more potent than resveratrol) that are structurally unrelated to resveratrol have been recently identified [2]. It will be interesting to characterise these small molecule sirtuin 1 inhibitors in immune regulation and type 1 diabetes mellitus treatment in mouse models of diabetes and in humans.

Acknowledgements We thank J. Borensztajn (Northwestern University) for technical support. This study was supported by NIH R01AI079056, R21AG028493 and a 'The Type I Diabetes Pathfinder Award' (DK083050) to D. Fang, and by grant R21AI68746 from National Institutes of Health (to H. Zaghouani). D. M. Tartar was supported by a life science fellowship from the University of Missouri, Columbia. This paper was supported by research funds for 2010 from Chonbuk National University (to S.-M. Lee). This work was also supported by the Northwestern University Interdepartmental ImmunoBiology Flow Cytometry Core Facility.

Duality of interest The authors declare that there is no duality of interest associated with this manuscript.

\section{References}

1. Schoppner A, Kindl H (1984) Purification and properties of a stilbene synthase from induced cell suspension cultures of peanut. J Biol Chem 259:6806-6811

2. Milne JC, Lambert PD, Schenk S et al (2007) Small molecule activators of SIRT1 as therapeutics for the treatment of type 2 diabetes. Nature 450:712-716

3. Bertelli AA, Das DK (2009) Grapes, wines, resveratrol, and heart health. J Cardiovasc Pharmacol 54:468-476

4. Bishayee A, Politis T, Darvesh AS (2010) Resveratrol in the chemoprevention and treatment of hepatocellular carcinoma. Cancer Treat Rev 36:43-53

5. Harikumar KB, Aggarwal BB (2008) Resveratrol: a multitargeted agent for age-associated chronic diseases. Cell Cycle 7:1020-1035

6. Das S, Das DK (2007) Anti-inflammatory responses of resveratrol. Inflamm Allergy Drug Targets 6:168-173

7. Imler TJ Jr, Petro TM (2009) Decreased severity of experimental autoimmune encephalomyelitis during resveratrol administration is associated with increased IL-17+IL-10+ T cells, CD4(-) IFNgamma + cells, and decreased macrophage IL-6 expression. Int Immunopharmacol 9:134-143

8. Singh NP, Hegde VL, Hofseth LJ, Nagarkatti M, Nagarkatti P (2007) Resveratrol (trans-3, 5, 4'-trihydroxystilbene) ameliorates experimental allergic encephalomyelitis, primarily via induction of apoptosis in $\mathrm{T}$ cells involving activation of aryl hydrocarbon receptor and estrogen receptor. Mol Pharmacol 72:1508-1521

9. Hsieh YH, Huang SS, Wei FC, Hung LM (2007) Resveratrol attenuates ischemia-reperfusion-induced leukocyte-endothelial cell adhesive interactions and prolongs allograft survival across the MHC barrier. Circ J 71:423-428

10. Wu SL, Yu L, Jiao XY, Meng KW, Pan CE (2006) The suppressive effect of resveratrol on protein kinase $\mathrm{C}$ theta in peripheral blood $\mathrm{T}$ lymphocytes in a rat liver transplantation model. Transplant Proc 38:3052-3054

11. Stefani M, Markus MA, Lin RC, Pinese M, Dawes IW, Morris BJ (2007) The effect of resveratrol on a cell model of human aging. Ann N Y Acad Sci 1114:407-418

12. Feige JN, Lagouge M, Canto C et al (2008) Specific SIRT1 activation mimics low energy levels and protects against diet-induced metabolic disorders by enhancing fat oxidation. Cell Metab 8:347-358

13. Elliott PJ, Jirousek M (2008) Sirtuins: novel targets for metabolic disease. Curr Opin Investig Drugs 9:371-378

14. Subbaramaiah K, Chung WJ, Michaluart P et al (1998) Resveratrol inhibits cyclooxygenase-2 transcription and activity in phorbol ester-treated human mammary epithelial cells. J Biol Chem 273:21875-21882

15. Borra MT, Smith BC, Denu JM (2005) Mechanism of human SIRT1 activation by resveratrol. J Biol Chem 280:17187-17195

16. Kaeberlein M, McDonagh T, Heltweg B et al (2005) Substratespecific activation of sirtuins by resveratrol. J Biol Chem 280:17038-17045

17. Kobayashi Y, Furukawa-Hibi Y, Chen C et al (2005) SIRT1 is critical regulator of FOXO-mediated transcription in response to oxidative stress. Int J Mol Med 16:237-243

18. Zhang J, Lee SM, Shannon S et al (2009) The type III histone deacetylase Sirt1 is essential for maintenance of T cell tolerance in mice. J Clin Invest 119:3048-3058

19. Lee JH, Song MY, Song EK et al (2009) Overexpression of SIRT1 protects pancreatic beta-cells against cytokine toxicity by suppressing the nuclear factor-kappaB signaling pathway. Diabetes 58:344-351

20. Tartar DM, VanMorlan AM, Wan X et al (2010) FoxP3+ RORgammat $+\mathrm{T}$ helper intermediates display suppressive function against autoimmune diabetes. J Immunol 184:3377-3385 
21. Chen A, Gao B, Zhang J et al (2009) The HECT-type E3 ubiquitin ligase AIP2 inhibits activation-induced T cell death by catalyzing EGR2 ubiquitination. Mol Cell Biol 29:5348-5356

22. Tanaka K, Nanbara S, Tanaka T, Koide H, Hayashi T (1988) Aminotransferase activity in the liver of diabetic mice. Diabetes Res Clin Pract 5:71-75

23. Liang SC, Tan XY, Luxenberg DP et al (2006) Interleukin (IL)-22 and IL-17 are coexpressed by Th17 cells and cooperatively enhance expression of antimicrobial peptides. J Exp Med 203:2271-2279

24. Sato K, Suematsu A, Okamoto K et al (2006) Th17 functions as an osteoclastogenic helper $\mathrm{T}$ cell subset that links $\mathrm{T}$ cell activation and bone destruction. J Exp Med 203:2673-2682

25. Yi T, Chen Y, Wang L et al (2009) Reciprocal differentiation and tissue-specific pathogenesis of Th1, Th2, and Th17 cells in graftvs-host disease. Blood 114:3101-3112

26. Bisikirska B, Colgan J, Luban J, Bluestone JA, Herold KC (2005) TCR stimulation with modified anti-CD3 mAb expands CD8+ T cell population and induces $\mathrm{CD} 8+\mathrm{CD} 25+$ Tregs. J Clin Invest 115:2904-2913

27. Rustenbeck I, Wienbergen A, Bleck C, Jorns A (2004) Desensitization of insulin secretion by depolarizing insulin secretagogues. Diabetes 53(Suppl 3):S140-S150

28. Marks BR, Nowyhed HN, Choi JY et al (2009) Thymic selfreactivity selects natural interleukin 17-producing $\mathrm{T}$ cells that can regulate peripheral inflammation. Nat Immunol 10:11251132

29. Reboldi A, Coisne C, Baumjohann D et al (2009) C-C chemokine receptor 6-regulated entry of TH-17 cells into the CNS through the choroid plexus is required for the initiation of EAE. Nat Immunol 10:514-523

30. Axtell RC, Steinman L (2009) Gaining entry to an uninflamed brain. Nat Immunol 10:453-455

31. Campbell JJ, Hedrick J, Zlotnik A, Siani MA, Thompson DA, Butcher EC (1998) Chemokines and the arrest of lymphocytes rolling under flow conditions. Science 279:381-384

32. Dieu MC, Vanbervliet B, Vicari A et al (1998) Selective recruitment of immature and mature dendritic cells by distinct chemokines expressed in different anatomic sites. J Exp Med 188:373-386

33. Power CA, Church DJ, Meyer A et al (1997) Cloning and characterization of a specific receptor for the novel CC chemokine MIP-3alpha from lung dendritic cells. J Exp Med 186:825-835
34. Baba M, Imai T, Nishimura $M$ et al (1997) Identification of CCR6, the specific receptor for a novel lymphocyte-directed CC chemokine LARC. J Biol Chem 272:14893-14898

35. Hieshima K, Imai T, Opdenakker $G$ et al (1997) Molecular cloning of a novel human $\mathrm{CC}$ chemokine liver and activationregulated chemokine (LARC) expressed in liver. Chemotactic activity for lymphocytes and gene localization on chromosome 2 . J Biol Chem 272:5846-5853

36. Hromas R, Gray PW, Chantry D et al (1997) Cloning and characterization of exodus, a novel beta-chemokine. Blood 89:3315-3322

37. Nikolic T, Geutskens SB, van Rooijen N, Drexhage HA, Leenen PJ (2005) Dendritic cells and macrophages are essential for the retention of lymphocytes in (peri)-insulitis of the nonobese diabetic mouse: a phagocyte depletion study. Lab Invest 85:487-501

38. Emamaullee JA, Davis J, Merani S et al (2009) Inhibition of Th17 cells regulates autoimmune diabetes in NOD mice. Diabetes 58:1302-1311

39. Nikoopour E, Schwartz JA, Huszarik K et al (2010) Th17 polarized cells from nonobese diabetic mice following mycobacterial adjuvant immunotherapy delay type 1 diabetes. J Immunol 184:4779-4788

40. Bending D, de la Pena H, Veldhoen $M$ et al (2009) Highly purified Th17 cells from BDC2.5NOD mice convert into Th1-like cells in NOD/SCID recipient mice. J Clin Invest 119:565-572

41. Martin-Orozco N, Chung Y, Chang SH, Wang YH, Dong C (2009) Th17 cells promote pancreatic inflammation but only induce diabetes efficiently in lymphopenic hosts after conversion into Th1 cells. Eur J Immunol 39:216-224

42. Marwaha AK, Crome SQ, Panagiotopoulos C et al (2010) Cutting edge: increased IL-17-secreting T cells in children with new-onset type 1 diabetes. J Immunol 185:3814-3818

43. Honkanen J, Nieminen JK, Gao R et al (2010) IL-17 immunity in human type 1 diabetes. J Immunol 185:1959-1967

44. Howitz KT, Bitterman KJ, Cohen HY et al (2003) Small molecule activators of sirtuins extend Saccharomyces cerevisiae lifespan. Nature 425:191-196

45. Pacholec M, Bleasdale JE, Chrunyk B et al (2010) SRT1720, SRT2183, SRT1460, and resveratrol are not direct activators of SIRT1. J Biol Chem 285:8340-8351

46. Juhasz B, Varga B, Gesztelyi R, Kemeny-Beke A, Zsuga J, Tosaki A (2010) Resveratrol: a multifunctional cytoprotective molecule. Curr Pharm Biotechnol 11:810-818 